\title{
烯烃连接的三嗪共轭多孔聚合物的合成及其可见光催化产氢性能
}

\author{
麻旺坪贺彦彦刘洪来* \\ (华东理工大学 化学工程联合国家重点实验室 化学与分子工程学院 上海 200237)
}

\begin{abstract}
摘要 近年来, 多孔有机光催化材料因其结构和能带易调控、比表面积大和密度小等优点而备受关注. 本工作以 Horner-Wadsworth-Emmons (HWE)反应为基础, 使用有机碱 $(t-\mathrm{BuOK}$ )作为催化剂, 在室温下制备了两例 $\mathrm{C}=\mathrm{C}$ 连接的三 嗪共轭多孔聚合物, 分别是由双取代的苯环和三甲基三嗪通过 $\mathrm{C}=\mathrm{C}$ 键连接的结构(TB-CPN)和由三苯胺和三甲基三嗪 通过 $\mathrm{C}=\mathrm{C}$ 键连接的结构 (TT-CPN), 将其用于光降解水产氢. 通过固体核磁和红外等结构表征, 证明 $\mathrm{C}=\mathrm{C}$ 的存在, 说 明结构的正确性. 然后, 在 $300 \mathrm{~W}$ 氙灯光源下分别将两个聚合物作为光催化剂进行裂解水产氢研究, 其中, TT-CPN 的 产氢速率达到了 $913.3 \mu \mathrm{mol} \cdot \mathrm{h}^{-1} \cdot \mathrm{g}^{-1}$, 相同条件下, TB-CPN 的产氢速率为 TT-CPN 的 $86 \%$. 此外, TT-CPN 在连续照射反 应 $25 \mathrm{~h}$ 后, 其光催化活性几乎没有降低, 表现出良好的光化学稳定性和可重复性. 本工作采用了一种低温、短反应时 间的方法来合成 $\mathrm{C}=\mathrm{C}$ 连接的共轭多孔聚合物, 用于可见光照射下光催化分解水制氢, 期望为多孔聚合物的设计合成 提供新的方案.
\end{abstract}

关键词＼cjkstart低温合成; 烯烃连接; 多孔聚合物; 光催化; 析氢

\section{Olefin-linked Conjugated Porous Networks and Their Visible-Light-Driven Hydrogen Evolution Performance}

\author{
Wangping Ma Yanyan He Honglai Liu* \\ (School of Chemistry and Molecular Engineering, State Key Laboratory of Chinese Chemical Engineering, \\ East China University of Science and Technology, Shanghai 200237, China)
}

\begin{abstract}
Global energy storage and environmental pollution have received increasing attention, and finding sustainable clean energy to replace fossil fuels has become an urgent issue. Visible-light-driven photocatalytic water splitting can not only obtain clean hydrogen energy, but also can store solar energy. Therefore, great efforts have been put into research and it has consequently developed rapidly. In recent years, different types of photocatalysts, such as inorganic materials, metal complexes, organic dyes and porous organic networks, have been extensively explored. Among these photocatalysts, conjugated porous networks (CPNs) have attracted much attention due to their adjustable structures, high specific surface area and high structural stability. The band gap and the specific surface area of materials is significant to photocatalytic performance. Hence, exploring appropriate band gap and porosity of materials is the main challenge, which can be achieved by changing the reaction methods and reactants to adjust the materials structure. In this article, unsubstituted olefin-linked $(\mathrm{C}=\mathrm{C}) \mathrm{CPNs}$ through Horner-Wadsworth-Emmons (HWE) reaction have been designed, which are the structure of disubstituted benzene and trimethyltriazine connected by $\mathrm{C}=\mathrm{C}$ bond $(\mathrm{TB}-\mathrm{CPN})$ and the structure of triphenylamine and trimethyltriazine connected by $\mathrm{C}=\mathrm{C}$ bond (TT-CPN) respectively. In this method, organic base $(t$-BuOK) was used as a catalyst, and finished the reaction at room temperature, which cost less energy. The entire synthesis procedure takes relatively short time, compared with other polymeric means costing $72 \mathrm{~h}$ or $48 \mathrm{~h}$. Firstly, the formation of olefin $(\mathrm{C}=\mathrm{C})$ bonds was characterized by Fourier transform infrared (FT-IR) spectroscopy. Next, the structure of the polymers was further confirmed by solid-state nuclear magnetic resonance. Nitrogen adsorption-desorption measurement was applied to examine the porosity of the polymers, it showed that TT-CPN has a larger specific surface area than TB-CPN. The band gap of TT-CPN and TB-CPN obtained by UV-Vis diffuse reflection spectra is 2.22 and $2.30 \mathrm{eV}$, respectively. After that, the two polymers were used as photocatalysts to investigate the visible-light-driven hydrogen evolution by water splitting. Of these, the porous polymer TT-CPN reveals a better photocatalytic behavior with a hydrogen evolution rate of $913.3 \mu \mathrm{mol} \bullet \mathrm{h}^{-1} \cdot \mathrm{g}^{-1}$. Under the same testing condition, the hydrogen evolution rate of TB-CPN was only $86 \%$ of TT-CPN. Besides, after TT-CPN was continuously irradiated under visible light for 25 $\mathrm{h}$, its photocatalytic activity barely decreased, showing good photochemical stability and repeatability. In a word, in this article, a method with low-temperature and short reaction time was developed to synthesize $\mathrm{C}=\mathrm{C}$ bond connected conjugated porous networks, and the polymers were used for efficient photocatalytic water splitting to produce hydrogen under visible light irradiation. This method may provide a new choice for the design and synthesis of porous polymers as photocatalysts. Keywords low-temperature synthesis; olefin-linkage; conjugated porous networks; photocatalysis; hydrogen evolution
\end{abstract}

*E-mail: hlliu@ecust.edu.cn; Tel.: 021-64252921

Received March 30, 2021; published June 15, 2021.

Supporting information for this article is available free of charge via the Internet at http://sioc-journal.cn.

Project supported by the National Natural Science Foundation of China (No. 91834301).

项目受国家自然科学基金(No. 91834301)资助. 


\section{1 引言}

能源与环境问题是当前人类面临的巨大挑战. 氢气 是一种公认的清洁能源, 而光催化制氢是有望实现将太 阳能转化为氢能的较为简单的方法. 然而, 开发出具备 高效光催化分解水性能的催化剂, 从而实现氢能的低成 本和大规模工业化生产仍然是一个重大挑战 ${ }^{[1-2]}$. 自从 Fujishima 和 Honda 在 1972 年利用 $\mathrm{TiO}_{2}$ 通过光电催化的 方式首次实现分解水制氢以来 ${ }^{[3]}$, 无机半导体光催化剂 已经得到了广泛的研究 ${ }^{[1-2,4-8]}$. 相比于无机光催化剂, 有机光催化剂结构和能带易于调控且成本较低 ${ }^{[9]}$, 更容 易得到良好的可见光催化活性. 共轭多孔聚合物作为一 种新型的有机光催化剂, 具有孔结构可调控、结构稳定 性高、比表面积大和可见光吸收性强等优点, 近年来受 到广泛的关注 ${ }^{[10-12]}$. 三嗪基共轭多孔聚合物因三嗪环具 有强烈的吸电子特性, 有助于光催化过程中电荷转换, 因此已被广泛探究用于光催化分解水制氢 [13-17]. 然而, 大多数此类聚合物通过极化的动态共价键(例如亚胺键) 连接, 在骨架上表现出相对较弱的稳定性, 严重阻碍了 实际应用. 设计烯烃 $(\mathrm{C}=\mathrm{C})$ 连接的三嗪基共轭多孔聚合
物是构建具有扩展 $\pi$ 共轭结构, 合成稳定性的结构的一 种非常直接且实用的方法.

Horner-Wadsworth-Emmons (HWE)反应 ${ }^{[18-19]}$ 是膦酸 酯基和醛基之间的化学反应，主要产生反式乙烯基. 反 应过程中提供了稳定的内鎓盐碳负离子，且反式乙烯基 的不可逆步骤的活化能较低, 因此从热力学和动力学上 分析, HWE 反应都有利于反式乙烯基的形成 ${ }^{[20]}$. 基于这 些前提，本工作采用 HWE 反应合成了两个具有稳定性 结构的聚合物 TB-CPN 和 TT-CPN(图 1). 这两个聚合物 作为催化剂在光催化分解水中得到了较好的产氢性能, TB-CPN 和 TT-CPN 在可见光下产氢的速率分别为 792.2 和 $913.3 \mu \mathrm{mol} \cdot \mathrm{h}^{-1} \cdot \mathrm{g}^{-1}$.

\section{2 结果与讨论}

\section{1 聚合物的结构及形貌分析}

通过固体核磁共振法(ss NMR), 傅里叶变换红外光 谱仪(FT-IR)和 $X$ 射线光电子能谱(XPS)对 TB-CPN 和 TT-CPN 的化学结构进行表征. TB-CPN 和 TT-CPN 的

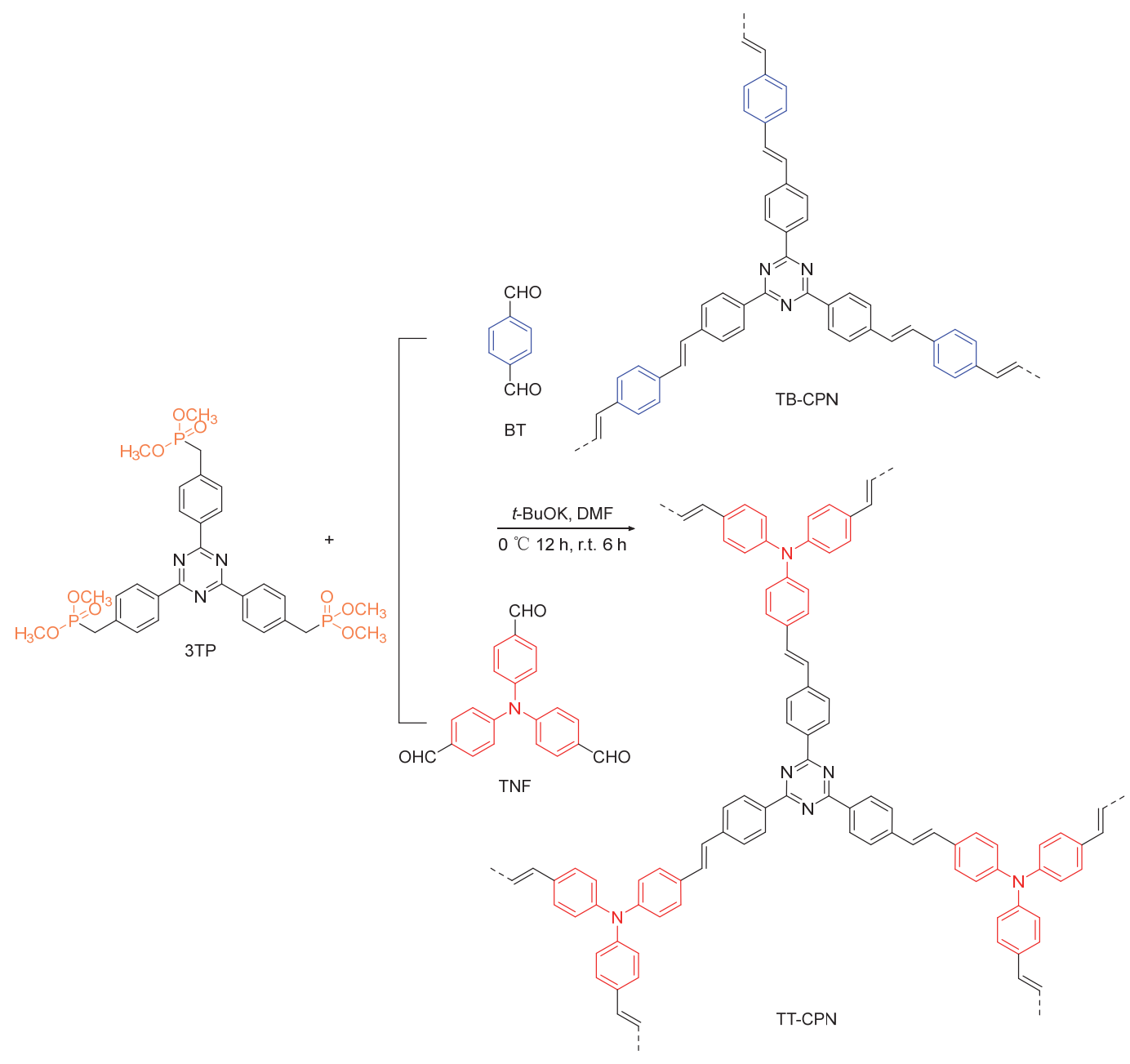

图 1 TB-CPN 和 TT-CPN 合成示意图

Figure 1 Synthesis schematic diagram of TB-CPN and TT-CPN 
Ss ${ }^{13} \mathrm{C}$ NMR 谱图如图 2(a)所示, 在化学位移约 $\delta 120$ 和 $\delta$ 140 的峰对应于 $\mathrm{C}=\mathrm{C}$ 上的碳峰, $\delta 127 \sim 136$ 处的重叠峰 归属于苯环上的芳香碳, $\delta 170$ 处的峰证实了框架中存 在三嗪环. 两个聚合物与对应单体的 FT-IR 图谱对比如 图 S2 所示, 对苯二甲醛(BT)和三(4-甲酰苯基)胺(TNF) 均在 $1650 \sim 1780 \mathrm{~cm}^{-1}$ 出现了强吸收峰, 即为醛基的特 征峰, 单体 $3 \mathrm{TP}$ 在 $1247 \mathrm{~cm}^{-1}$ 左右出现了 $\mathrm{P}=\mathrm{O}$ 特征 峰 ${ }^{21]}$, 而两个聚合物均未表现出醛基和 $\mathrm{P}=\mathrm{O}$ 特征峰, 表明聚合反应进行完全. 两个聚合物的傅里叶变换红外 光谱如图 2(b)所示, 在峰值约 $1606 \mathrm{~cm}^{-1}$ 处出现 $-\mathrm{C}=$ $\mathrm{C}$ 一伸缩振动峰, 在约 $978 \mathrm{~cm}^{-1}$ 处则对应于反式 $\mathrm{HC}=$ $\mathrm{CH}$ 拉伸振动, 表明了这些多孔聚合物中存在反式烯烃 部分 ${ }^{[22-23]}$; 在 $1500 \sim 1510 \mathrm{~cm}^{-1}, 1360 \sim 1370 \mathrm{~cm}^{-1}$ 和 $790 \sim 800 \mathrm{~cm}^{-1}$ 均出现三嗪环的特征峰. 此外, 图 2(c)所 示为 TB-CPN 和 TT-CPN 的高分辨 C $1 \mathrm{~s}$ XPS 谱图, 结合 能为 $284.2 \mathrm{eV}$ 处的 $\mathrm{C}=\mathrm{C}(\mathrm{C}-\mathrm{C})^{[24]}$ 和 $286.4 \mathrm{eV}$ 处的 $\mathrm{C}=\mathrm{N}(\mathrm{C}-\mathrm{N})^{[25]}$, 说明了两者化学结构中苯环和三嗪环 基团的存在. 因此, 以上多项表征均说明了合成得到的 TB-CPN 和 TT-CPN 结构准确性, 为下一步的性能表征 奠定了基础.
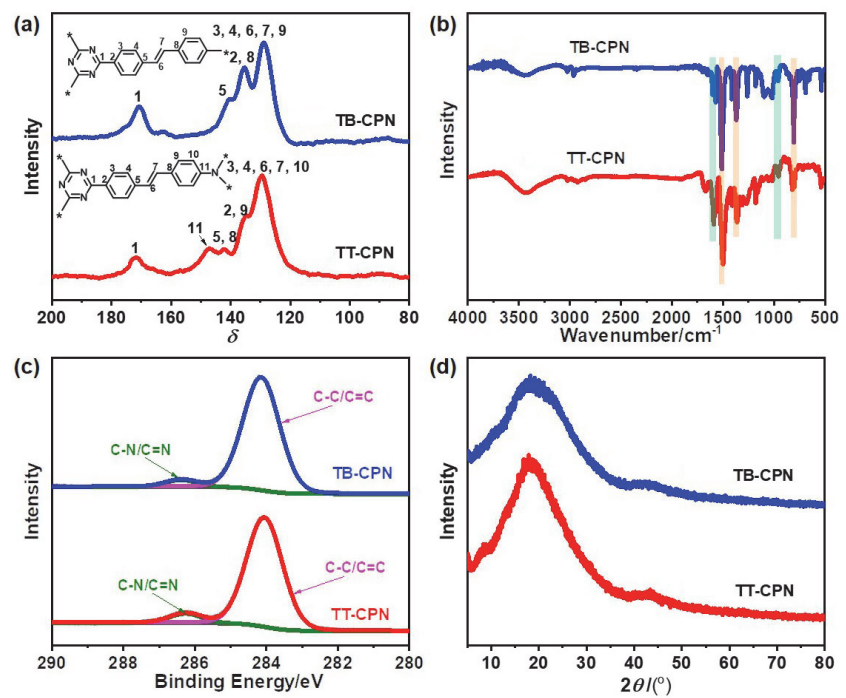

图 2 TB-CPN 和 TT-CPN 的(a) $\mathrm{ss}^{13} \mathrm{C}$ NMR 谱图, (b) 傅里叶变换红外 光谱图, (c) C $1 \mathrm{~s} \mathrm{X}$ 射线光电子能谱和(d) 粉末 $X$ 射线衍射光谱

Figure 2 (a) ss NMR spectra, (b) FT-IR spectra, (c) C 1s XPS spectra and (d) PXRD spectra of TB-CPN and TT-CPN

聚合物 TB-CPN 和 TT-CPN 经溶剂测试不溶于甲 醇、乙醇、乙酸乙酯、丙酮、二氯甲烷、甲苯、四氢呋 喃、 $N, N$ 二 二甲基甲酰胺(DMF) 等常见有机溶剂, 证明 TB-CPN 和 TT-CPN 具有良好的溶剂稳定性. 图 3 展示 了 TB-CPN 和 TT-CPN 在氮气氛围下的热重分析结果. TB-CPN 和 TT-CPN 质量减少 $10 \%$ 时的温度分别为 567 和 $585{ }^{\circ} \mathrm{C}$, 加热到 $800{ }^{\circ} \mathrm{C}$ 仍有 $65 \%$ 以上剩余材料, 说明 了 TB-CPN 和 TT-CPN 均具有非常好的热稳定性.

通过对材料的粉末 $\mathrm{X}$ 射线衍射(PXRD)光谱分析, 如图 2(d)所示, 在 $2 \theta=20^{\circ}$ 左右两个聚合物均出现了宽
的衍射峰，表明了这两个聚合物的无定形性质. 如图 4 所示, 通过扫描电子显微镜(SEM) 和透射电子显微镜 (TEM)观察所制备的多孔聚合物的形貌. SEM 图像观察 到材料的微观形貌为不规则块状结构; TEM 图像中的明 暗对比可以明显观察到两个材料的孔结构, 表明了这两 个聚合物样品均为无定形的多孔结构.

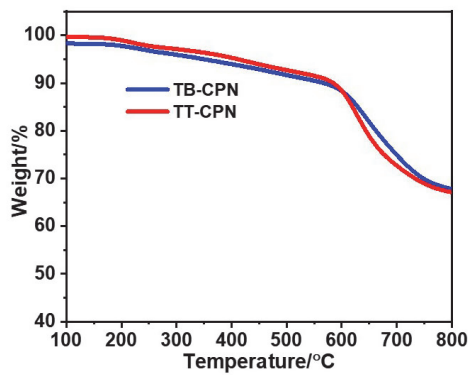

图 3 TB-CPN 和 TT-CPN 的热重分析图

Figure 3 Thermogravimetric analysis of TB-CPN and TT-CPN
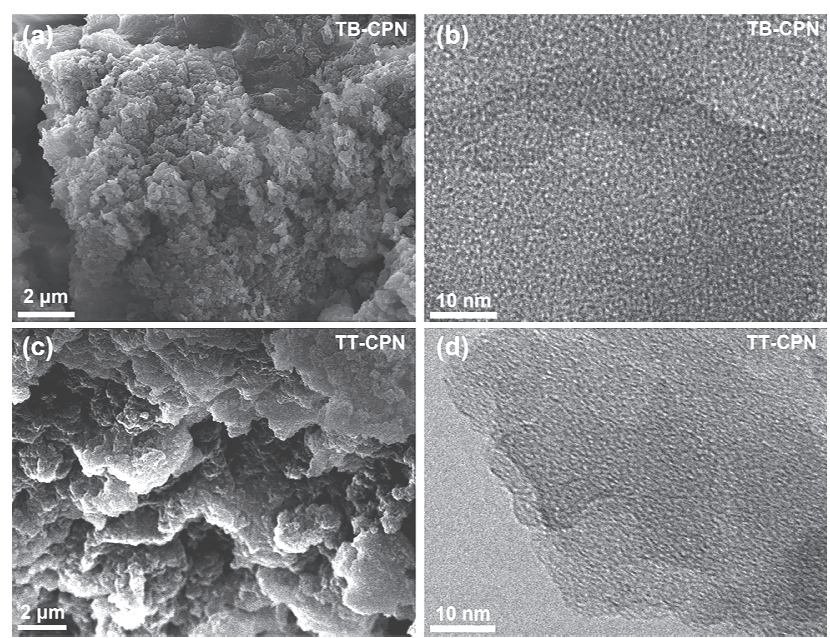

图 4 TB-CPN (a)和 TT-CPN (c)的 SEM 图; TB-CPN (b)和 TT-CPN (d) 的 TEM 图

Figure 4 SEM images of (a) TB-CPN and (c) TT-CPN; TEM images of (b) TB-CPN and (d) TT-CPN

通过测试 $77 \mathrm{~K}$ 下 $\mathrm{N}_{2}$ 吸附-脱附等温线表征 TB-CPN 和 TT-CPN 的多孔性质, 如图 5 所示. TB-CPN 和 TT-CPN 在标准状态下相对压力较低 $\left(P / P_{0}<0.01\right)$ 时气体 吸附曲线较陡, 根据 IUPAC 分类标准, 可判断 TB-CPN 和 TT-CPN 均为 I 型氮吸附等温线, 表明两个聚合物均 具有微孔性质. 如图 S4(a), (b) 所示, 在 $P / P_{0}=0.05 \sim 0.3$ 范围内分别做 TB-CPN 和 TT-CPN 的 BET (BrunauerEmmett-Teller)曲线，计算得 TB-CPN 和 TT-CPN 的比表 面积分别为 103 和 $620 \mathrm{~m}^{2} \cdot \mathrm{g}^{-1}$. 两个聚合物在相对较低 的压力下, 吸附和解吸曲线之间都观察到滞后现象, 这 可能是由于含有柔性乙烯基连接的聚合物网络的形变 和膨胀所致 ${ }^{[26]}$. 根据非局部密度泛函理论(NLDFT)计算 得到两个聚合物的孔径分布, TB-CPN 的孔径主要分布 在 $1.7 \mathrm{~nm}$ 和 $2.8 \mathrm{~nm}$ (图 5c), 说明该材料不仅存在微孔结 
构, 还具有介孔结构; 如图 5(d)所示, TT-CPN 的孔径主 要分布在 $1.7 \mathrm{~nm}$, 表明存在大量微孔结构.
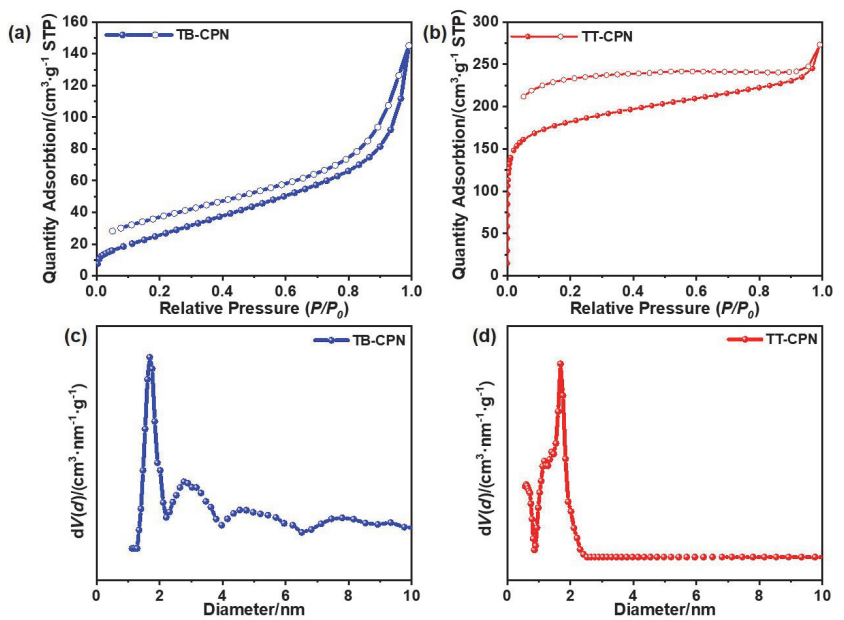

图 5 (a) TB-CPN 和(b) TT-CPN 的 $\mathrm{N}_{2}$ 吸附-解吸等温曲线; (c) TB-CPN 和(d) TT-CPN 的孔径分布图

Figure 5 Nitrogen adsorption and desorption isotherm curves of (a) TB-CPN and (b) TT-CPN; and pore size distribution diagrams of (c) TB-CPN and (d) TT-CPN

\section{2 材料的光学性能分析}

使用紫外可见漫反射光谱(DRS UV-Vis)研究了材 料的光物理性质, TB-CPN 和 TT-CPN 这两个固态多孔 聚合物均显示出宽泛的吸收带, 涵盖了紫外到可见光区 域，见图 6(a). 同时由 Kubelka-Munk 变换得到 TB-CPN 和 TT-CPN 的光学带隙分别为 $2.30 \mathrm{eV}$ 和 $2.22 \mathrm{eV}$, 如图 6(b). 两种聚合物的固体荧光光谱如图 6(c)所示, 在波
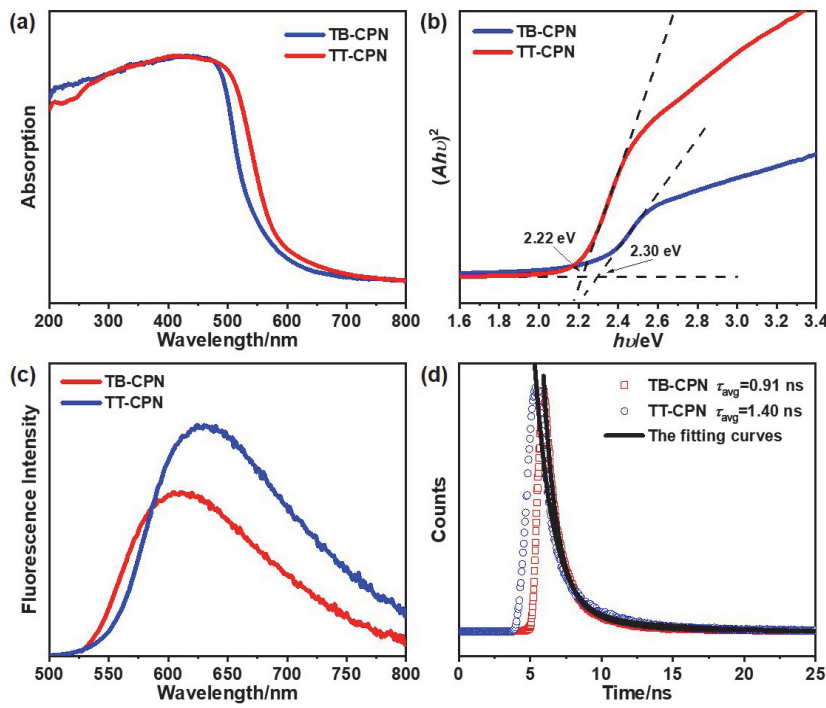

图 6 TB-CPN 和 TT-CPN 的 (a) 紫外-可见吸收光谱, (b) 由 Kubelka-Munk 变换的反射光谱确定的聚合物带隙, (c)稳态荧光光谱 和(d) 时间分辨瞬态光致发光衰减

Figure 6 (a) UV-Vis absorption spectra, (b) band gaps of the polymers determined from the Kubelka-Munk transformed reflectance spectra, (c) photoluminescence spectra and (d) time-resolved transient photoluminescence decay of TB-CPN and TT-CPN
长为 $365 \mathrm{~nm}$ 的光激发下, 两个聚合物均在可见光区域 发出荧光. 聚合物 TB-CPN 和 TT-CPN 的最大发射波长 分别为 $600 \mathrm{~nm}$ 和 $621 \mathrm{~nm}$. 基于时间分辨的菼光光谱如 图 6(d)所示, 分别对 TB-CPN 和 TT-CPN 苂光衰减曲线 的下降区域进行指数拟合 ${ }^{[27]}$, 得出了重组电荷载流子 的平均辐射寿命 $\left(\tau_{\mathrm{avg}}\right)$, TB-CPN, TT-CPN 的 $\tau_{\mathrm{avg}}$ 值分别为 0.91 和 $1.40 \mathrm{~ns}$. TT-CPN 的荧光寿命更长, 表明 TT-CPN 有较低的载流子重组率，载流子的分离效率较高.

如图 7 所示, 通过紫外光电子能谱(UPS)确定电离 电势(即价带能 $E_{\mathrm{v}}$ ), 通过从激发能 $(21.22 \mathrm{eV}$ )减去 UPS 光谱的宽度计算得出 TB-CPN, TT-CPN 的价带能分别为 4.75 和 $5.12 \mathrm{eV}$. 因此, 根据 $E_{\mathrm{v}}-E_{\mathrm{opt}}$ 估计导带能量 $E_{\mathrm{c}}$ 为 2.45 和 $2.90 \mathrm{eV}$.
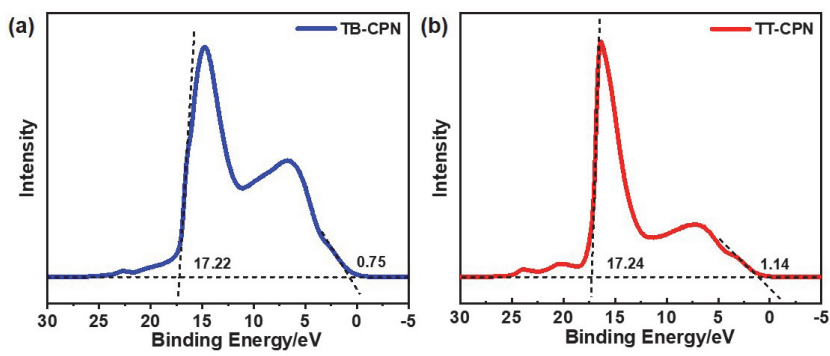

图 7 (a) TB-CPN 和(b) TT-CPN 的紫外光电子能谱图 Figure 7 UPS spectra of (a) TB-CPN and (b) TT-CPN

\section{3 可见光光催化水分解测试分析}

在可见光 $(\lambda>420 \mathrm{~nm})$ 的照射下, 两个 $\mathrm{Pt}$ 负载的多 孔聚合物均表现出一定产氢活性, 产氢的性能列于表 1 和图 8 中。这两个聚合物的析氢速率(HERs)如下: TB-CPN $\left(792.2 \mu \mathrm{mol} \cdot \mathrm{h}^{-1} \cdot \mathrm{g}^{-1}\right)<$ TT-CPN $\left(913.3 \mu \mathrm{mol} \bullet \mathrm{h}^{-1} \bullet\right.$ $\left.\mathrm{g}^{-1}\right)$. 产氢性能与文献报道的同类型的材料相比表现较 好 ${ }^{[15,28-32]}$. 此外, 如图 8(c)所示, TT-CPN 在可见光下连 续照射反应 $25 \mathrm{~h}$ 后，其光催化活性几乎没有降低，表现 出良好的光化学稳定性. 图 S5(a)给出了 TT-CPN 通过光 化学沉积负载 Pt 后的 TEM 图, 可以观察到 Pt 在材料表 面分散较为均一. 图 S5(b)则展示了 TT-CPN 在循环 $25 \mathrm{~h}$ 后的 TEM 图, 从中可以看出循环后 $\mathrm{Pt}$ 颗粒出现了少量 的团聚现象.

表 1 聚合物的孔隙率数据和光学性能

Table 1 Porosity data and electrochemical properties of the polymers

\begin{tabular}{ccccc}
\hline Sample & $\begin{array}{c}S_{\mathrm{BET}}{ }^{a} / \\
\left(\mathrm{m}^{2} \cdot \mathrm{g}^{-1}\right)\end{array}$ & $\begin{array}{c}E_{\mathrm{opt}}{ }^{b} / \\
\mathrm{eV}\end{array}$ & $\begin{array}{c}E_{\mathrm{v}}{ }^{c} / E_{\mathrm{c}}{ }^{d} \\
(\mathrm{~V} \mathrm{vs} . \mathrm{SHE})\end{array}$ & $\begin{array}{c}\mathrm{HER}_{\mathrm{vis}}{ }^{e} / \\
\left(\mu \mathrm{mol}^{-1} \cdot \mathrm{g}^{-1}\right)\end{array}$ \\
\hline TB-CPN & 103 & 2.30 & $4.75 / 2.45$ & 792.2 \\
TT-CPN & 620 & 2.22 & $5.12 / 2.90$ & 913.3 \\
\hline
\end{tabular}

${ }^{a}$ Surface area calculated from the $\mathrm{N}_{2}$ adsorption-desorption isotherm using BET theory. ${ }^{b}$ Optical band gaps derived from the absorption edges. ${ }^{c}$ Evaluated by UPS spectra. ${ }^{d}$ Calculated from $E_{\mathrm{v}}-E_{\text {opt }}{ }^{e}$ Reaction conditions: $25 \mathrm{mg}$ polymer, $25 \mathrm{~mL} 0.2 \mathrm{~mol} \cdot \mathrm{L}^{-1}$ ascorbic acid (AA) aqueous solution, $1 \mathrm{~mL} 2$ $\mathrm{mg} \cdot \mathrm{mL}^{-1} \mathrm{H}_{2} \mathrm{PtCl}_{6} \cdot 6 \mathrm{H}_{2} \mathrm{O}(w(\mathrm{Pt})=3 \%), 300 \mathrm{~W}$ Xe light $(15 \mathrm{~A}-14 \mathrm{~V}) \mathrm{Xe}$ light, $\lambda \geqslant 420 \mathrm{~nm}$. 

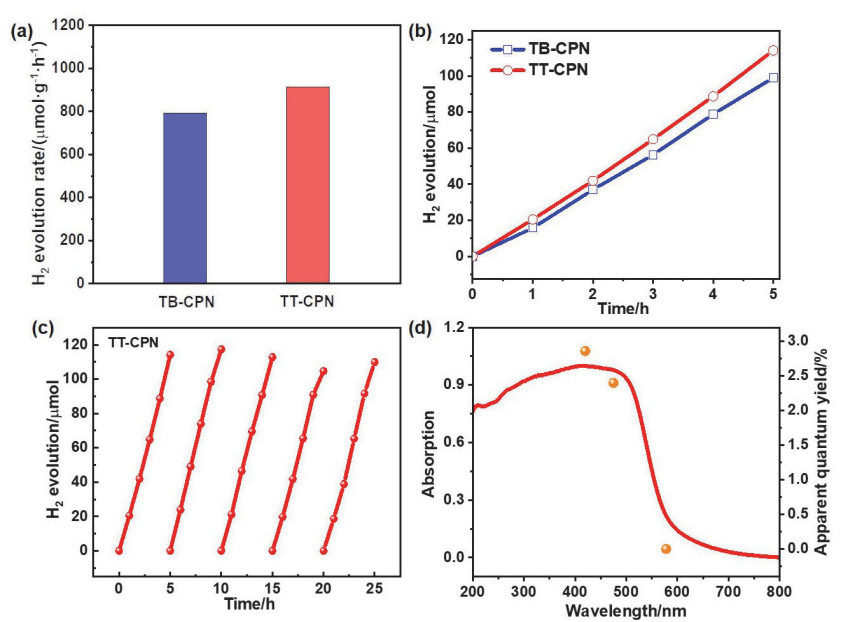

图 8 TB-CPN 和 TT-CPN 的(a) 光催化水分解产氢速率, (b) 光催化水 分解产氢量与时间的关系, (c) TT-CPN 的光催化产氢稳定性测试, (d) TT-CPN 在不同波长下 $(\lambda=420 、 475$ 和 $578 \mathrm{~nm})$ 的光催化表观量子产 率和紫外漫反射光谱对比

Figure 8 (a) Photocatalytic $\mathrm{H}_{2}$ evolution rates, (b) time course of $\mathrm{H}_{2}$ evolution from water by the TB-CPN and TT-CPN, (c) stability and reusability tests using TT-CPN as a photocatalyst under visible-light irradiation, (d) wavelength-dependent AQY and DRS spectrum of TT-CPN $(\lambda=420,475$ and $578 \mathrm{~nm})$

如图 8(d)所示, 以 TT-CPN 为例, 使用 $300 \mathrm{~W} \mathrm{Xe}$ 光 源在不同的滤光片下 $(\lambda=420 \mathrm{~nm} 、 475 \mathrm{~nm}$ 和 $578 \mathrm{~nm})$ 照 射，测试该材料在不同波长下的表观量子产率(AQY), 在波长 $420 \mathrm{~nm}$ 时 $\mathrm{AQY}$ 可达到 $2.86 \%$.

\section{3 结论}

使用含三嗪基的 3TP 单体和不同的含醛基官能团 衍生物作为关键单体, 通过 HWE 反应成功在室温和相 对较短的反应时间下合成 $\mathrm{C}=\mathrm{C}$ 连接的共轭微孔聚合物 TB-CPN 和 TT-CPN, 此类材料能在可见光照射下实现 催化裂解水制氢. 在波长 $420 \mathrm{~nm}$ 以上的可见光照射下, TB-CPN 和 TT-CPN 均可以产生氢气, 它们的产氢效率 分别达到了 792.2 和 $913.3 \mu \mathrm{mol} \cdot \mathrm{h}^{-1} \cdot \mathrm{g}^{-1}$. TT-CPN 的产氢 效率明显高于 TB-CPN, 这可能归因于以下原因：(1) TT-CPN 拥有更高的比表面积; (2) TT-CPN 有更窄的光 学带隙, 可以在更宽的区域内收集可见光. (3)相比于 TB-CPN, 氮掺杂的 TT-CPN 具有更多的光催化活性位 点, 有利于电子与质子的结合, 更有利于光催化产氢过 程的进行.

总之, 本工作使用一种低温、短反应时间的方法来 合成未取代烯烃连接的三嗪共轭多孔聚合物, 用于可见 光照射下高效光催化分解水制氢, 并且材料具有良好的 稳定性和可重复性, 为此类光催化聚合物提供了一种合 理的合成方案, 为合理开发用于太阳能转换的更为高效 经济的材料提供了一个新的方法.

\section{4 实验部分}

\section{1 材料合成}

\subsection{1 单体 3TP 合成}

$3 T P$ 的合成步骤参考文献 ${ }^{[33-36]}$, 具体分为两步(图 S1), 分别为:

(1) 在 $250 \mathrm{~mL}$ 烧瓶中, 将 $11.0 \mathrm{~g}$ 对甲基苯甲腈溶于 $80 \mathrm{~mL}$ 超干二氯甲烷中. 在冰浴下将 $25.0 \mathrm{~g}$ 三氟甲磺酸 缓慢加入该溶液中. 将混合物在室温下搅拌 $12 \mathrm{~h}$ 后缓慢 滴入冰水淬灭, 接着缓慢滴入适量氨水中和. 滤出沉淀 物, 用水和丙酮分别洗涤 3 次. 然后将所得固体用甲苯 重结晶, 固体样品在 $80{ }^{\circ} \mathrm{C}$ 烘箱中干燥过夜, 得到白色 针状固体 3T, 产率: $68 \%$.

(2) 在 $100 \mathrm{~mL}$ 圆底烧瓶中, 将 $3.51 \mathrm{~g} 3 \mathrm{~T}, 5.34 \mathrm{~g}$ $N$-溴丁二酰胺(NBS)和 $0.3 \mathrm{~g}$ 过氧化苯甲酰(BPO)溶于 50 $\mathrm{mL}$ 超干氯苯中, 并在 $110{ }^{\circ} \mathrm{C}$ 加热 $7 \mathrm{~h}$. 过滤混合物, 得 到黄色滤液, 减压蒸馏除去滤液中的溶剂. 将得到残余 物溶于 $10 \mathrm{~mL}$ 亚磷酸三甲酯中回流 $9 \mathrm{~h}$. 减压蒸馏除去 过量的亚磷酸三甲酯. 将粗产物通过硅胶柱色谱法纯 化, 将固体样品在 $80{ }^{\circ} \mathrm{C}$ 烘箱中干燥过夜, 得到淡黄色 固体 3TP, 产率: $55 \%$.

\subsection{2 聚合物的合成}

聚合物的合成路线如图 1 所示.

(1) TB-CPN 合成在 $50 \mathrm{~mL}$ Schlenk 烧瓶中, 氮气保 护下将 $100 \mathrm{mg} 3 \mathrm{TP}$ 和 $32 \mathrm{mg}$ 对苯二甲醛(98\%, 北京华 威锐科化工有限公司)溶解在 $10 \mathrm{~mL}$ 超干 DMF (99.8\%, 北京百灵威科技有限公司)中. 待混合物溶解均匀后, 在冰水浴中, 快速加入 $107 \mathrm{mg}$ 叔丁醇钾 $(t$-BuOK, $99 \%$, 上海泰坦科技股份有限公司). 然后将混合物继续置于 冰浴中搅拌 $12 \mathrm{~h}$. 之后撤去冰浴, 使混合物慢慢升温至 室温, 并在室温下继续摚拌 $6 \mathrm{~h}$. 过滤得到不溶的共轭 聚合物, 分别用 DMF $(50 \mathrm{~mL} \times 3)$, 四氢呋喃 $(50$ $\mathrm{mL} \times 3)$, 乙醇 $(50 \mathrm{~mL} \times 3)$ 和二氯甲烷 $(50 \mathrm{~mL} \times 3)$ 洗涤, 以除去未反应完全的单体或催化剂. 将得到的固体在 $80{ }^{\circ} \mathrm{C}$ 下真空干燥过夜, 得到黄色粉末状固体, 产率: $85 \%$.

(2) TT-CPN 的合成步骤与 TB-CPN 类似. TT-CPN: 橘黄色粉末状固体, 产率: $88 \%$.

\section{2 光催化测试}

首先在光催化制氢系统中, 装入 $25 \mathrm{mg}$ 聚合物粉末, $25 \mathrm{~mL} 0.2 \mathrm{~mol} \cdot \mathrm{L}^{-1}$ 抗坏血酸(AA)水溶液. 将所得混合物 超声处理 $30 \mathrm{~min}$, 以获得分散良好的光催化剂悬浮液. 然后将得到的悬浮液加入到光催化反应器当中. 为了进 一步提高光催化性能, 向上述悬浮液中加入 $1 \mathrm{~mL}$ $\mathrm{H}_{2} \mathrm{PtCl}_{6} \cdot 6 \mathrm{H}_{2} \mathrm{O} \quad(w(\mathrm{Pt})=3 \%)$, 在光照条件下, $\mathrm{H}_{2} \mathrm{PtCl}_{6} \cdot 6 \mathrm{H}_{2} \mathrm{O}$ 被还原成单质 $\mathrm{Pt}$ 沉积在光催化剂的表面 上以充当助催化剂, 通过等离子体发射光谱仪(ICP)测 得 TT-CPN 的实际 Pt 负载量为 $w(\mathrm{Pt})=1.6 \%$. 然后, 将系 
统用 $\mathrm{Ar}$ 脱氧 $30 \mathrm{~min}$ 以在辐照前除去空气. 使用 $300 \mathrm{~W}$ 全波长氙灯 $(15 \mathrm{~A}-14 \mathrm{~V})$ 作为照射光源. 通过气相色谱仪 (GC-2014C, Shimadzu, 以 $\mathrm{Ar}$ 作为载气)测量 $\mathrm{H}_{2}$ 的逸出 量.

\section{3 表观量子产率(AQY)计算}

以 $300 \mathrm{~W}$ 单色 $\mathrm{Xe}$ 光源 $(\lambda=420 \mathrm{~nm} 、 475 \mathrm{~nm}$ 和 578 $\mathrm{nm}$ )照射, 进行了 AQY 计算. $\mathrm{H}_{2}$ 还原的表观量子产率 (AQY)由下式 ${ }^{[15,37]}$ 计算:

$$
\begin{aligned}
\eta_{\mathrm{AQY}} & =2 \times \mathrm{H}_{2} \text { 产量 } / \text { 入射光子数 } \times 100 \% \\
& =2 \times\left(M N_{\mathrm{A}} h c\right) /(S P t \lambda) \times 100 \%
\end{aligned}
$$

其中, $M$ 为 $\mathrm{H}_{2}$ 的物质的量, $N_{\mathrm{A}}$ 为阿伏伽德罗常数 $\left(6.022 \times 10^{23} \mathrm{~mol}^{-1}\right), h$ 为普朗克常量 $\left(6.626 \times 10^{-34} \mathrm{~J} \bullet \mathrm{s}\right), c$ 为光速 $\left(3 \times 10^{8} \mathrm{~m} \cdot \mathrm{s}^{-1}\right), S$ 为辐照面积 $\left(\mathrm{cm}^{2}\right), P$ 为辐照光强 $\left(\mathrm{W} \cdot \mathrm{cm}^{-2}\right), t$ 是光反应时间 $(\mathrm{s}), \lambda$ 是单色光的波长 $(\mathrm{m})$.

\section{References}

[1] Zou, X.; Zhang, Y. Chem. Soc. Rev. 2015, 44, 5148.

[2] Rahman, M. Z.; Kibria, M. G.; Mullins, C. B. Chem. Soc. Rev. 2020 , 49, 1887.

[3] Fujishima, A.; Honda, K. Nature 1972, 238, 37.

[4] Wang, X.; Maeda, K.; Thomas, A.; Takanabe, K.; Xin, G.; Carlsson, J. M.; Domen, K.; Antonietti, M. Nature Mater. 2009, 8, 76.

[5] Xing, J.; Chen, J. F.; Li, Y. H.; Yuan, W. T.; Zhou, Y.; Zheng, L. R.; Wang, H. F.; Hu, P.; Wang, Y.; Zhao, H. J.; Wang, Y.; Yang, H. G. Chemistry 2014, 20, 2138.

[6] Liu, J.; Liu, Y.; Liu, N.; Han, Y.; Zhang, X.; Huang, H.; Lifshitz, Y.; Lee, S. T.; Zhong, J.; Kang, Z. Science 2015, 347, 970.

[7] Li, C.; Chen, F.; Ye, L.; Li, W.; Yu, H.; Zhao, T. Acta Chim. Sinica 2020,78, 1448. (李宸, 陈凤华, 叶丽, 李伟, 于晗, 赵䇛, 化学学 报, 2020, 78, 1448).

[8] Guo, Y.; Li, Y.; Wang, C.; Long, R.; Xiong, Y. Acta Chim. Sinica 2019, 77, 520. (郭宇, 李燕瑞, 王成名, 龙冉, 熊宇杰, 化学学报, 2019, 77, 520).

[9] Xu, Z.-Y.; Luo, Y.; Wang, H.; Zhang, D.-W.; Li, Z.-T. Chin. J. Org. Chem. 2020, 40, 3777. (徐子悦, 罗驿, 王辉, 张丹维, 黎占亭, 有 机化学, 2020, 40, 3777).

[10] Zhang, G.; Lan, Z. A.; Wang, X. Angew. Chem. Int. Ed. 2016, 55, 15712 .

[11] Dai, C.; Liu, B. Energy Environ. Sci. 2020, 13, 24.

[12] Ma, X.; Wang, H.; Cheng, J.; Cheng, H.; Wang, L.; Wu, X.; Xu, H. Chin. J. Chem. 2021, 39, 1079.

[13] Banerjee, T.; Gottschling, K.; Savasci, G.; Ochsenfeld, C.; Lotsch, B. V. ACS Energy Lett. 2018, 3, 400 .
[14] Wang, X.; Chen, L.; Chong, S. Y.; Little, M. A.; Wu, Y.; Zhu, W. H.; Clowes, R.; Yan, Y.; Zwijnenburg, M. A.; Sprick, R. S.; Cooper, A. I. Nature Chem. 2018, 10, 1180.

[15] Yu, K.; Bi, S.; Ming, W.; Wei, W.; Zhang, Y.; Xu, J.; Qiang, P.; Qiu, F.; Wu, D.; Zhang, F. Polym. Chem. 2019, 10, 3758.

[16] Kong, D.; Han, X.; Xie, J.; Ruan, Q.; Windle, C. D.; Gadipelli, S.; Shen, K.; Bai, Z.; Guo, Z.; Tang, J. ACS Catal. 2019, 9, 7697.

[17] Huang, W.; He, Q.; Hu, Y.; Li, Y. Angew. Chem. Int. Ed. 2019, 58, 8676.

[18] Horner, L.; Hoffmann, H.; Wipp, H. G. Chem. Ber. 1958, 91, 61.

[19] Wadsworth., W. S.; Emmons, W. D. J. Am. Chem. Soc. 1961, 83, 1733.

[20] Ando, K. J. Org. Chem. 1998, 63, 8411.

[21] Shi, M.; Yamamoto, K.; Okamoto, Y.; Takamuku, S. Phosphorus Sulfur 1991, 60, 1.

[22] Xu, J.; He, Y.; Bi, S.; Wang, M.; Yang, P.; Wu, D.; Wang, J.; Zhang, F. Angew. Chem. Int. Ed. 2019, 58, 12065.

[23] Bi, S.; Thiruvengadam, P.; Wei, S.; Zhang, W.; Zhang, F.; Gao, L.; Xu, J.; Wu, D.; Chen, J. S.; Zhang, F. J. Am. Chem. Soc. 2020, 142, 11893.

[24] Zhuang, X.; Zhao, W.; Zhang, F.; Cao, Y.; Liu, F.; Bi, S.; Feng, X. Polym. Chem. 2016, 7, 4176.

[25] Zhu, X.; Tian, C.; Mahurin, S. M.; Chai, S. H.; Wang, C.; Brown, S.; Veith, G. M.; Luo, H.; Liu, H.; Dai, S. J. Am. Chem. Soc. 2012, 134, 10478.

[26] Jiang, J.-X.; Trewin, A.; Adams, D. J.; Cooper, A. I. Chem. Sci. 2011, 2, 1777.

[27] Aitchison, C. M.; Sachs, M.; Little, M. A.; Wilbraham, L.; Brownbill, N. J.; Kane, C. M.; Blanc, F.; Zwijnenburg, M. A.; Durrant, J. R.; Sprick, R. S.; Cooper, A. I. Chem. Sci. 2020, 11, 8744.

[28] Vyas, V. S.; Haase, F.; Stegbauer, L.; Savasci, G.; Podjaski, F.; Ochsenfeld, C.; Lotsch, B. V. Nat. Commun. 2015, 6, 8508.

[29] Xu, Y.; Mao, N.; Zhang, C.; Wang, X.; Zeng, J.; Chen, Y.; Wang, F.; Jiang, J. Appl. Catal. B 2018, 228, 1.

[30] Mothika, V. S.; Sutar, P.; Verma, P.; Das, S.; Pati, S. K.; Maji, T. K. Chemistry 2019, 25, 3867.

[31] Sachs, M.; Cha, H.; Kosco, J.; Aitchison, C. M.; Francàs, L.; Corby, S.; Chiang, C.-L.; Wilson, A. A.; Godin, R.; Fahey-Williams, A.; Cooper, A. I.; Sprick, R. S.; McCulloch, I.; Durrant, J. R. J. Am. Chem. Soc. 2020, 142, 14574.

[32] Wu, Q.; Zhang, C.; Sun, K.; Jiang, H.-L. Acta Chim. Sinica 2020 , 78, 688. (吴浅耶, 张晨曦, 孙康, 江海龙, 化学学报, 2020, 78, $688)$

[33] Jiang, Y.; Wang, Y.; Hua, J.; Tang, J.; Li, B.; Qian, S.; Tian, H. Chem. Commun. 2010, 46, 4689.

[34] Jiang, Y.; Wang, Y.; Wang, B.; Yang, J.; He, N.; Qian, S.; Hua, J. Chem. Asian J. 2011, 6, 157.

[35] Lin, Z.; Emge, T. J.; Warmuth, R. Chemistry 2011, 17, 9395.

[36] Pradhan, B.; Pathak, S. K.; Gupta, R. K.; Gupta, M.; Pal, S. K.; Achalkumar, A. S. J. Mater. Chem. C 2016, 4, 6117.

[37] Huang, T.; Lin, X.; Liu, Y.; Zhao, J.; Lin, H.; Xu, Z.; Zhong, S.; Zhang, C.; Wang, X.; Fu, X.; Long, J. ChemSusChem 2020, 13, 672.

(Cheng, B.) 